\title{
Prediction of survival after neoadjuvant chemotherapy for breast cancer by evaluation of tumor-infiltrating lymphocytes and residual cancer burden
}

(a) CrossMark

Yuka Asano ${ }^{1}$, Shinichiro Kashiwagi ${ }^{*}$ (D), Wataru Goto ${ }^{1}$, Koji Takada ${ }^{1}$, Katsuyuki Takahashi ${ }^{2}$, Takaharu Hatano ${ }^{3}$, Satoru Noda', Tsutomu Takashima' ${ }^{1}$ Naoyoshi Onoda' ${ }^{1}$ Shuhei Tomita ${ }^{2}$, Hisashi Motomura ${ }^{3}$, Masahiko Ohsawa ${ }^{4}$, Kosei Hirakawa ${ }^{1}$ and Masaichi Ohira ${ }^{1}$

\begin{abstract}
Background: The tumor immune environment not only modulates the effects of immunotherapy, but also the effects of other anticancer drugs and treatment outcomes. These immune responses can be evaluated with tumor-infiltrating lymphocytes (TILS), which has frequently been verified clinically. On the other hand, residual cancer burden (RCB) evaluation has been shown to be a useful predictor of survival after neoadjuvant chemotherapy (NAC). In this study, RCB and TILs evaluations were combined to produce an indicator that we have termed "RCB-TILs", and its clinical application to NAC for breast cancer was verified by subtype-stratified analysis.

Methods: A total of 177 patients with breast cancer were treated with NAC. The correlation between RCB and TILs evaluated according to the standard method, and prognosis, including the efficacy of NAC, was investigated retrospectively. The RCB and TILs evaluations were combined to create the "RCB-TILs". Patients who were RCB-positive and had high TILs were considered RCB-TILs-positive, and all other combinations were RCB-TILs-negative.

Results: On multivariable analysis, being RCB-TILs-positive was an independent factor for recurrence after NAC in all patients $(p<0.001$, hazard ratio $=0.048)$, triple-negative breast cancer (TNBC) patients $(p=0.018$, hazard ratio $=0.041)$, HER2-positive breast cancer (HER2BC) patients $(p=0.036$, hazard ratio $=0.134)$, and hormone receptor-positive breast cancer (HRBC) patients $(p=0.002$, hazard ratio $=0.081$ ).
\end{abstract}

Conclusions: The results of the present study suggest that RCB-TILs is a significant predictor for breast cancer recurrence after NAC and may be a more sensitive indicator than TILS alone.

Keywords: Residual cancer burden, Tumor-infiltrating lymphocytes, Neoadjuvant chemotherapy, Breast cancer, Predictive marker

\footnotetext{
*Correspondence: spqv9ke9@view.ocn.ne.jp

${ }^{1}$ Department of Surgical Oncology, Asahi-machi, Abeno-ku, Osaka 545-8585,

Japan

Full list of author information is available at the end of the article
} 


\section{Background}

Treatment with neoadjuvant chemotherapy (NAC) increases the rate of breast-conserving surgery and reduces the risk of postoperative recurrence in patients with resectable breast cancer [1-4]. The main purposes of NAC are to facilitate tumor regression, improve breast conservation rates, evaluate therapeutic effects, and establish therapeutic strategies based on the evaluation results $[1,5,6]$. Recently, NAC has required tailoring, particularly by exploring biomarkers using genetic approaches or establishing therapeutic strategies based on the response to early treatment. Although previous studies have described the prediction of survival after NAC by means of the pathological complete response ( $\mathrm{pCR}$ ) rate, tumorinfiltrating lymphocytes (TILs), and residual cancer burden (RCB), none of these have yet come into use in actual clinical practice [7-12].

Cancer cells have various gene abnormalities that allow them to proliferate spontaneously and survive, but the surrounding environment (cancer microenvironment) also influences cancer cells and is involved in the intrinsic characteristics of cancer [13]. The tumor immune environment not only influences the effects of immunotherapy but also the effects of other anticancer drugs and treatment outcomes $[1,14]$. Thus, the importance of inhibiting and improving the tumor immune microenvironment is now recognized. TILs are regarded as an indicator for monitoring such immune responses, and studies have found that they are prognostic factors and predictors of response to treatment in a range of types of cancer $[15,16]$. A large amount of evidence has now been reported for the clinical relevance of the morphological evaluation of TILs in breast cancer, and the subject is now attracting attention [9, 15-18]. We have previously reported the clinical validity and utility of the evaluation of TILs in NAC [19].

RCB evaluation has been shown to be a useful predictor of survival after NAC $[11,12]$. RCB after NAC is calculated by a method developed by Symmans and colleagues at the University of Texas MD Anderson Cancer Center [11]. One study that used this calculation method for the analysis of survival after NAC found that, for the triple-negative breast cancer (TNBC) and hormone receptor-positive breast cancer (HRBC) subtypes, RCB evaluation was useful for predicting longterm survival [12].

TILs are also believed to be useful markers for predicting response to treatment in the TNBC and human epidermal growth factor receptor-2 (HER2)-positive breast cancer (HER2BC) subtypes, which are associated with high levels of immune activity [20]. We therefore hypothesized that combining the evaluation of TILs with that of RCB might provide a sensitive indicator that is also capable of predicting survival in HRBC. In this study, RCB and TILs evaluations were combined to produce an indicator that we have termed "RCB-TILs", and its clinical application to NAC for breast cancer was verified by subtype-stratified analysis.

\section{Methods \\ Patient background}

This study was conducted at Osaka City University Graduate School of Medicine, Osaka, Japan, according to the Reporting Recommendations for Tumor Marker prognostic Studies (REMARK) guidelines and a retrospectively written research, pathological evaluation, and statistical plan. Written, informed consent was obtained from all patients. This research conformed to the provisions of the Declaration of Helsinki of 2013. The study protocol was approved by the Ethics Committee of Osaka City University (\#926).

A total of 177 patients with resectable, early-stage breast cancer diagnosed as stage IIA (T1, N1, M0 or T2, N0, M0), IIB (T2, N1, M0 or T3, N0, M0), or IIIA (T12, N2, M0 or T3, N1-2, M0) were treated with NAC between 2007 and 2013. Tumor stage and T and N factors were stratified based on the TNM Classification of Malignant Tumors, UICC Seventh Edition [21]. Our previous reports have also used the same patient population and the present study, but it was the study of the significance of CD8 /FOXP3 ratio or androgen receptor $[19,22]$. Breast cancer was confirmed histologically by core needle biopsy and staged by systemic imaging studies using computed tomography (CT), ultrasonography (US), and bone scintigraphy. Breast cancer was classified into subtypes according to the immunohistochemical expressions of estrogen receptor (ER), progesterone receptor (PgR), HER2, and Ki67. Based on their immunohistochemical expression profiles, tumors are categorized into immunophenotypes: luminal A (ER+ and/or PgR+, HER2-, Ki67-low); luminal $\mathrm{B}(\mathrm{ER}+$ and/or PgR+, HER2+) (ER+ and/or PgR+, HER2-, Ki67-high), HER2-enriched (HER2BC) (ER-, PgR-, and HER2+); and TNBC (negative for ER, PgR, and HER2) [23]. In this study, luminal A and luminal B were considered hormone receptor-positive breast cancer (HRBC).

All patients received a standardized protocol of NAC consisting of four courses of FEC100 $\left(500 \mathrm{mg} / \mathrm{m}^{2}\right.$ fluorouracil, $100 \mathrm{mg} / \mathrm{m}^{2}$ epirubicin, and $500 \mathrm{mg} / \mathrm{m}^{2}$ cyclophosphamide) every 3 weeks, followed by 12 courses of $80 \mathrm{mg} / \mathrm{m}^{2}$ paclitaxel administered weekly [24, 25]. Fortyfive patients had HER2-positive breast cancer and were given additional weekly $(2 \mathrm{mg} / \mathrm{kg})$ or tri-weekly $(6 \mathrm{mg} / \mathrm{kg})$ trastuzumab during paclitaxel treatment [26]. All patients underwent chemotherapy as outpatients. Therapeutic antitumor effects were assessed according to the Response Evaluation Criteria in Solid Tumors (RECIST) criteria [27]. Patients underwent mastectomy or breast-conserving surgery after NAC. The pathological effect of chemotherapy 
was assessed for resected primary tumors after NAC. Pathological complete response (pCR) was defined as the complete disappearance of the invasive components of the lesion with or without intraductal components, including in the lymph nodes, according to the National Surgical Adjuvant Breast and Bowel Project (NSABP) B-18 protocol [1]. All patients who underwent breast-conserving surgery underwent postoperative radiotherapy to the remnant breast. The standard postoperative adjuvant therapy for the subtype concerned was administered.

Overall survival (OS) time was the period from the initiation of NAC to the time of death from any cause. Disease-free survival (DFS) was defined as freedom from all local, loco-regional, and distant recurrences. All patients were followed-up by physical examination every 3 months, US every 6 months, and CT and bone scintigraphy annually. The median follow-up period was 3.4 years (range, 0.6-6.0 years) for the assessment of OS and 3.1 years (range, 0.1-6.0 years) for DFS. The primary end point of this study was DFS, and the secondary endpoint was OS and $\mathrm{pCR}$ rate.

\section{Histopathological evaluation of TILs}

Histopathological assessment of predictive factors was performed on core needle biopsy (CNB) specimens at the time of the breast cancer diagnosis. In this study, TILs were evaluated in the same method as our previous studies [28]. Histopathological parameters examined included nuclear grade, histological type, presence of TILs, and correlations of these parameters with intrinsic subtypes and pCR.

Histopathologic analysis of the percentage of TILs was evaluated on a single full-face hematoxylin and eosin (HE)-stained tumor section using criteria described by Salgado et al. [29]. TILs were defined as the infiltrating lymphocytes within tumor stroma and were expressed by the proportion of the field investigated, and the number of TILs in stroma surrounding the stained cancer cells was quantitatively measured in each field under 400-times magnification [30, 31]. The areas of in situ carcinoma and crush artifacts were not included. Proportional scores of 3, 2, 1, and 0 were given if the area of stroma containing lymphoplasmacytic infiltration around invasive tumor cell nests comprised $>50 \%,>10-50 \%, \leq 10 \%$, and $0 \%$, respectively. A score of $\geq 2$ was considered positive for TILs, whereas scores of 1 and 0 were considered negative. Histopathologic evaluation of TILs was jointly performed by two breast pathologists, who were blinded to clinical information, including treatment allocation and outcomes.

\section{Histopathological evaluation of RCB}

The RCB was calculated using the Residual Cancer Burden Calculator on the website of the MD Anderson Cancer Center [11]. This automatically calculates the $\mathrm{RCB}$ on the basis of data on the primary tumor (primary tumor bed area, overall cancer cellularity, and percentage of cancer that is in situ disease) and lymph node metastasis (number of positive lymph nodes and diameter of largest metastasis). The RCB is categorized into one of three classes: minimal residual disease (RCB-I), moderate residual disease (RCB-II), or extensive residual disease (RCB-III). Since RCB-I is considered to have a better prognosis than RCB-II and RCB-III, RCB-I was considered positive, and RBC-II and RCB-III were considered negative.

Table 1 Correlation between clinicopathological features and RCB-TILs in 177 breast cancers

\begin{tabular}{|c|c|c|c|}
\hline \multirow[t]{2}{*}{ Parameters } & \multicolumn{2}{|c|}{ RCB-TILs in all breast cancers $(n=177)$} & \multirow[t]{2}{*}{$\mathrm{p}$ value } \\
\hline & Positive $(n=112)$ & Negative $(n=65)$ & \\
\hline \multicolumn{4}{|l|}{ Age at operation } \\
\hline$\leq 56$ & $52(46.4 \%)$ & $35(53.9 \%)$ & \\
\hline$>56$ & $60(53.6 \%)$ & $30(46.1 \%)$ & 0.341 \\
\hline \multicolumn{4}{|l|}{ Menopause } \\
\hline Pre-menopausal & $44(39.3 \%)$ & $28(43.1 \%)$ & \\
\hline Post-menopausal & $68(60.7 \%)$ & $37(56.9 \%)$ & 0.621 \\
\hline \multicolumn{4}{|l|}{ Tumor size } \\
\hline$\leq 2 \mathrm{~cm}$ & $19(17.0 \%)$ & $5(7.7 \%)$ & \\
\hline$>2 \mathrm{~cm}$ & $93(83.0 \%)$ & $60(92.3 \%)$ & 0.082 \\
\hline \multicolumn{4}{|l|}{ Lymph node status } \\
\hline Negative & $27(24.1 \%)$ & $14(21.5 \%)$ & \\
\hline Positive & $85(75.9 \%)$ & $51(78.5 \%)$ & 0.696 \\
\hline \multicolumn{4}{|l|}{ Nuclear grade } \\
\hline 1,2 & $81(72.3 \%)$ & $56(86.2 \%)$ & \\
\hline 3 & $31(27.7 \%)$ & $9(13.8 \%)$ & 0.034 \\
\hline \multicolumn{4}{|l|}{ Ki67 } \\
\hline$\leq 14 \%$ & $36(32.1 \%)$ & $38(58.5 \%)$ & \\
\hline$>14 \%$ & $76(67.9 \%)$ & $27(41.5 \%)$ & 0.001 \\
\hline \multicolumn{4}{|l|}{ Intrinsic subtype } \\
\hline TNBC & 49 (43.8\%) & $12(16.0 \%)$ & \\
\hline non-TNBC & $63(56.2 \%)$ & $53(84.0 \%)$ & 0.001 \\
\hline \multicolumn{4}{|l|}{ Intrinsic subtype } \\
\hline HER2BC & $26(23.2 \%)$ & $10(15.4 \%)$ & \\
\hline non- HER2BC & $86(76.8 \%)$ & 55 (84.6\%) & 0.212 \\
\hline \multicolumn{4}{|l|}{ Intrinsic subtype } \\
\hline HRBC & 37 (33.0\%) & $43(66.2 \%)$ & \\
\hline non-HRBC & 75 (67.0\%) & $22(33.8 \%)$ & $<0.001$ \\
\hline \multicolumn{4}{|l|}{ Pathological response } \\
\hline $\mathrm{pCR}$ & $58(51.8 \%)$ & $9(13.8 \%)$ & \\
\hline non-pCR & $54(48.2 \%)$ & $56(86.2 \%)$ & $<0.001$ \\
\hline
\end{tabular}

$R C B$ residual cancer burden, TILs tumor-infiltrating lymphocytes, TNBC triplenegative breast cancer, $H E R 2 B C$ human epidermal growth factor receptor 2enriched breast cancer, $H R B C$ hormone receptor-positive breast cancer, $p C R$ pathological complete response 


\section{RCB-TILs evaluation}

The RCB and TILs evaluations were combined to create the "RCB-TILs". Patients who were RCB-I-positive and had positive TILs were considered RCB-TILs-positive, and all other combinations were RCB-TILs-negative.

\section{Statistical analysis}

Statistical analysis was performed using the SPSS version 19.0 statistical software package (IBM, Armonk, NY, USA). The associations between TILs, RCB-TILs, and clinicopathological variables were examined using $X^{2}$ tests. Multivariable analysis of pCR was carried out using a binary logistic regression model. The Kaplan-Meier method was used to estimate DFS and OS, and the results were compared between groups with log-rank tests. A Cox proportional hazards model was used to compute univariable and multivariable hazards ratios (HR) for the study parameters with 95\% confidence intervals (c.i.), and a backward stepwise method was used for variable selection in multivariable analyses. A $p$ value $<0.05$ was considered significant. Cutoff values for different biomarkers included in this study were chosen before statistical analysis.

\section{Results}

RCB-TILs and clinicopathological investigation

Of the patients who underwent NAC, 112 (63.3\%) were RCB-TILs-positive, and 65 (36.7\%) were negative. RCBTILs-positive patients had a significantly higher nuclear grade $(p=0.034)$, higher Ki67 value $(p=0.001)$, higher proportion of TNBC $(p=0.001)$, lower proportion of HRBC $(p<0.001)$, and a higher $\mathrm{pCR}$ rate $(p<0.001)$ (Table 1). A further investigation within each subtype was performed. Among the 61 patients with TNBC, RCB-TILs-positive patients had a significantly higher $\mathrm{pCR}$ rate $(p=0.023)$, whereas among HER2BC patients, RCB-TILs-positive patients had a significantly lower pCR rate $(p=0.004)$. In HRBC patients, RCB-TILspositive patients had a significantly higher nuclear grade $(p=0.004)$, higher Ki67 value $(p=0.024)$, and higher pCR rate $(p=0.007)$ (Table 2$)$.

\section{Analysis of survival according to RCB-TILs}

Survival was analyzed according to RCB-TILs. DFS after NAC was significantly longer for RCB-TILs-positive patients than for RCB-TILs-negative patients in all

Table 2 Correlations between RCB-TILs and clinicopathological parameters in 61 triple-negative, 36 HER2-positive, and 80 hormone receptor-positive breast cancers

\begin{tabular}{|c|c|c|c|c|c|c|c|c|c|}
\hline \multirow[t]{2}{*}{ Parameters } & \multicolumn{2}{|c|}{$\operatorname{TNBC}(n=61)$} & \multirow[t]{2}{*}{$p$ value } & \multicolumn{2}{|c|}{ HER2BC $(n=36)$} & \multirow[t]{2}{*}{$p$ value } & \multicolumn{2}{|c|}{$\operatorname{HRBC}(n=80)$} & \multirow[t]{2}{*}{$p$ value } \\
\hline & $\begin{array}{l}\text { Positive } \\
(n=49)\end{array}$ & $\begin{array}{l}\text { Negative } \\
(n=12)\end{array}$ & & $\begin{array}{l}\text { Positive } \\
(n=26)\end{array}$ & $\begin{array}{l}\text { Negative } \\
(n=10)\end{array}$ & & $\begin{array}{l}\text { Positive } \\
(n=37)\end{array}$ & $\begin{array}{l}\text { Negative } \\
(n=43)\end{array}$ & \\
\hline \multicolumn{10}{|l|}{ Age at operation } \\
\hline$\leq 56$ & $23(46.9 \%)$ & $5(41.7 \%)$ & & $12(46.2 \%)$ & $4(40.0 \%)$ & & $17(45.9 \%)$ & $26(60.5 \%)$ & \\
\hline$>56$ & $26(53.1 \%)$ & 7 (58.3\%) & 0.743 & $14(53.8 \%)$ & $6(60.0 \%)$ & 0.519 & $20(50.1 \%)$ & 17 (39.5\%) & 0.194 \\
\hline \multicolumn{10}{|l|}{ Menopause } \\
\hline Pre-menopausal & $17(34.7 \%)$ & $5(41.7 \%)$ & & $11(42.3 \%)$ & $3(30.0 \%)$ & & $16(43.2 \%)$ & $20(46.5 \%)$ & \\
\hline Post-menopausal & $32(65.3 \%)$ & $7(58.3 \%)$ & 0.652 & $15(57.7 \%)$ & 7 (70.0\%) & 0.389 & $21(56.8 \%)$ & $23(53.5 \%)$ & 0.770 \\
\hline \multicolumn{10}{|l|}{ Tumor size } \\
\hline$\leq 2 \mathrm{~cm}$ & $7(14.3 \%)$ & $0(0.0 \%)$ & & $5(19.2 \%)$ & $1(10.0 \%)$ & & 7 (18.9\%) & $4(9.3 \%)$ & \\
\hline$>2 \mathrm{~cm}$ & $42(85.7 \%)$ & $12(100.0 \%)$ & 0.197 & $21(80.8 \%)$ & $9(90.0 \%)$ & 0.456 & $30(81.1 \%)$ & 39 (90.7\%) & 0.179 \\
\hline \multicolumn{10}{|l|}{ Lymph node status } \\
\hline Negative & $9(18.4 \%)$ & $2(16.7 \%)$ & & $8(30.8 \%)$ & $3(30.0 \%)$ & & $10(27.0 \%)$ & 9 (20.9\%) & \\
\hline Positive & $40(81.6 \%)$ & $10(83.3 \%)$ & 0.630 & $18(69.2 \%)$ & $7(70.0 \%)$ & 0.647 & $27(73.0 \%)$ & 34 (79.1\%) & 0.353 \\
\hline \multicolumn{10}{|l|}{ Nuclear grade } \\
\hline 1,2 & $37(75.5 \%)$ & $7(58.3 \%)$ & & $19(73.1 \%)$ & $9(90.0 \%)$ & & $25(67.6 \%)$ & 40 (93.0\%) & \\
\hline 3 & $12(24.5 \%)$ & $5(41.7 \%)$ & 0.234 & 7 (26.9\%) & $1(10.0 \%)$ & 0.269 & $12(32.4 \%)$ & $3(7.0 \%)$ & 0.004 \\
\hline \multicolumn{10}{|l|}{ Ki67 } \\
\hline$\leq 14 \%$ & $13(26.5 \%)$ & $5(41.7 \%)$ & & $10(38.5 \%)$ & 7 (70.0\%) & & $13(35.1 \%)$ & $26(60.5 \%)$ & \\
\hline$>14 \%$ & $36(73.5 \%)$ & $7(58.3 \%)$ & 0.303 & $16(61.5 \%)$ & $3(30.0 \%)$ & 0.090 & $24(64.9 \%)$ & 17 (39.5\%) & 0.024 \\
\hline \multicolumn{10}{|l|}{ Pathological response } \\
\hline $\mathrm{pCR}$ & $26(53.1 \%)$ & $2(16.7 \%)$ & & $9(34.6 \%)$ & $9(90.0 \%)$ & & $15(40.5 \%)$ & $6(14.0 \%)$ & 0.007 \\
\hline non-pCR & 23 (46.9\%) & 10 (83.3\%) & 0.023 & $17(65.4 \%)$ & $1(10.0 \%)$ & 0.004 & $22(59.5 \%)$ & 37 (86.0\%) & \\
\hline
\end{tabular}

$R C B$ residual cancer burden, TILs tumor-infiltrating lymphocytes, TNBC triple-negative breast cancer, HER2BC human epidermal growth factor receptor 2-enriched breast cancer, $H R B C$ hormone receptor-positive breast cancer, $p C R$ pathological complete response 
patients $(p<0.001, \log$-rank), TNBC patients $(p<0.001$, log-rank), HER2BC patients ( $p=0.007$, log-rank), and HRBC patients ( $p=0.026$, log-rank) (Fig. 1a-d). Overall survival was significantly longer for RCB-TILs-positive patients than for RCB-TILs-negative patients in all patients $(p=0.005, \log$-rank $)$ and TNBC patients $(p<0.001$, logrank), but the difference was not significant for HER2BC patients $(p=0.585$, log-rank) or HRBC patients $(p=0.128$, log-rank) (Additional file 1: Figure S1A-D).

Univariable analysis of patients with high TILs found that this contributed significantly to prolonging DFS in all patients $(p=0.022$, HR $=0.420)$, TNBC patients $(p=0.004, \mathrm{HR}=0.177)$, and HER2BC patients $(p=0.026, \mathrm{HR}=0.123)$. For HRBC patients, however, high TILs did not contribute to survival $(p=0.990, \mathrm{HR}=0.992)$. Being RCB-TILs-positive, however, contributed significantly to prolonging DFS in all patients $(p<0.001$, $\mathrm{HR}=0.181)$, TNBC patients $(p<0.001, \mathrm{HR}=0.099)$, HER2BC patients $(\mathrm{p}=0.026, \mathrm{HR}=0.123)$, and HRBC patients $(p=0.039, \mathrm{HR}=0.258)$ (Table 3, Fig. 2a-d).

Receiver operating characteristic (ROC) analysis showed that, for all breast cancer patients, the results for the RCBTILs [area under the curve (AUC): 0.700] were better than those for the TILs (AUC: 0.606) and RCB (AUC: 0.538) (Fig. 3a-d). An analysis by subtype also found similar results for TNBC patients (AUC: TILs $=0.703, \mathrm{RCB}=0.624$, RCB-TILs $=0.768)($ Fig. 3e-h), HER2BC patients (AUC: TILs $=0.681, \mathrm{RCB}=0.539, \mathrm{RCB}-\mathrm{TILs}=0.687)($ Fig. $4 \mathrm{a}-\mathrm{d})$, and HRBC patients (AUC: TILs $=0.501, \mathrm{RCB}=0.622$, RCB-TILs $=0.650)($ Fig. 4e-h).

On multivariable analysis, high TILs was an independent factor contributing to prolonging DFS in all patients $(p=0.029, \mathrm{HR}=4.785), \mathrm{TNBC}$ patients $(p=0.023$, $\mathrm{HR}=0.243)$, and HER2BC patients $(p=0.036, \mathrm{HR}=0.134)$. For HRBC patients, however, no contribution to survival ( $p=0.949, \mathrm{HR}=1.044)$ was observed. Being RCB-TILspositive was an independent factor for recurrence after NAC in all patients $(p<0.001, \mathrm{HR}=0.048)$, TNBC patients $(p=0.018, \mathrm{HR}=0.041)$, HER2BC patients $(p=0.036, \mathrm{HR}=0.134)$, and HRBC patients $(p=0.002$, $\mathrm{HR}=0.081)$ (Table 3).

\section{Discussion}

The definition of pCR after NAC is based on tumor infiltration or non-infiltration and the status of the axillary lymph nodes [32]. DFS is clearly improved for patients

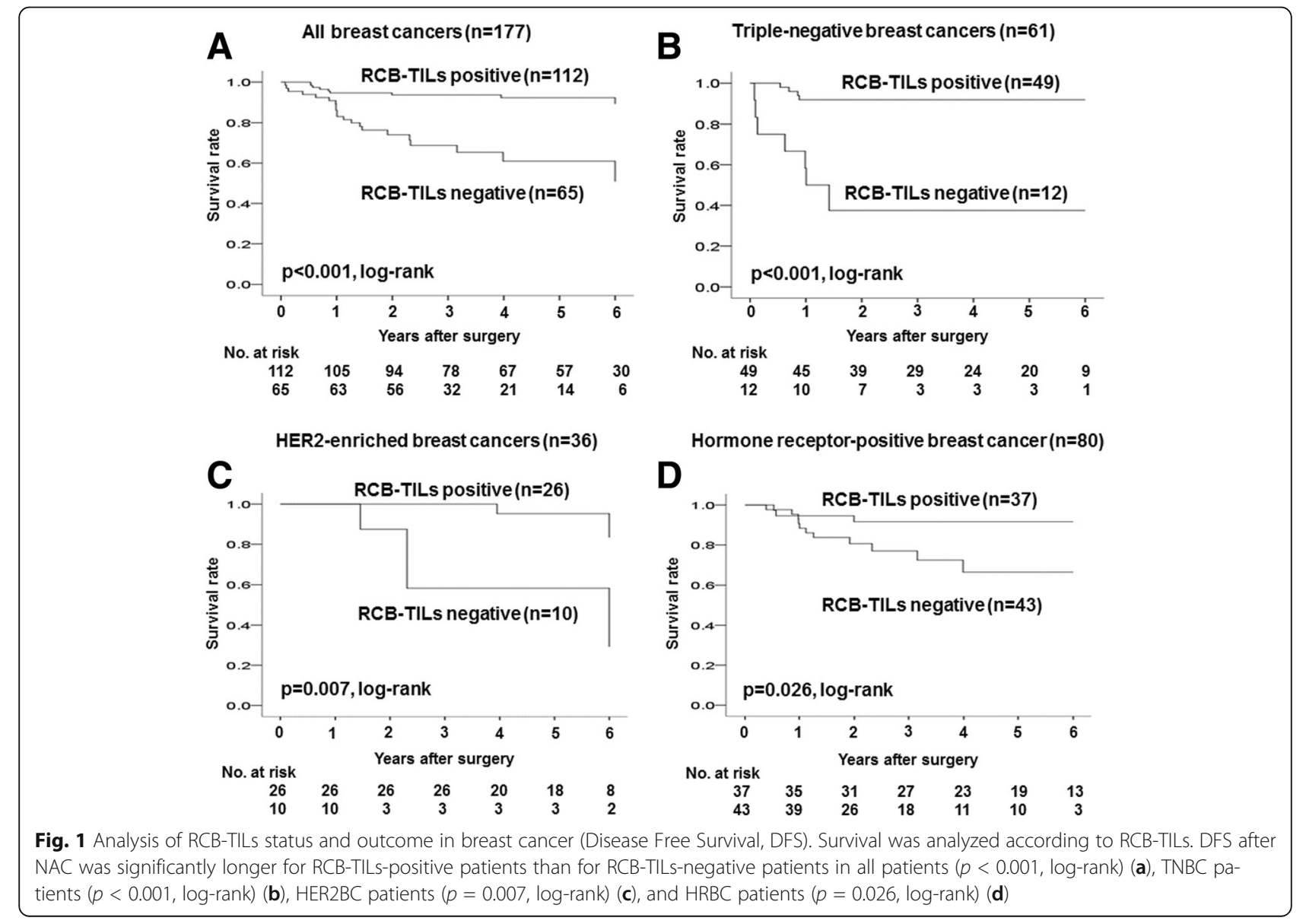


Table 3 Univariable and multivariable analysis with respect to disease-free survival in breast cancer subtypes

\begin{tabular}{|c|c|c|c|c|c|c|c|}
\hline \multirow{2}{*}{ Parameter } & & \multicolumn{3}{|c|}{ Univariable analysis } & \multicolumn{3}{|c|}{ Multivariable analysis } \\
\hline & & Hazard ratio & 95\% c.i. & $p$ value & Hazard ratio & $95 \%$ c.i. & $p$ value \\
\hline \multicolumn{8}{|l|}{ All breast cancers $(n=177)$} \\
\hline Age & $\leq 56$ vs $>56$ & 0.809 & $0.395-1.657$ & 0.561 & & & \\
\hline Menopause & Pre- vs Post- & 0.840 & $0.408-1.731$ & 0.637 & & & \\
\hline Tumor size (cm) & $\leq 2$ vs $>2$ & 1.062 & $0.370-3.045$ & 0.911 & & & \\
\hline Lymph node status & Negative vs Positive & 4.157 & $0.990-17.456$ & 0.052 & & & \\
\hline Nuclear grade & $1-2$ vs 3 & 1.025 & $0.440-2.389$ & 0.954 & & & \\
\hline Ki67 (\%) & $\leq 14$ vs $>14$ & 0.649 & $0.316-1.331$ & 0.238 & & & \\
\hline Intrinsic subtype & TNBC vs non-TNBC & 1.213 & $0.577-2.550$ & 0.611 & & & \\
\hline Intrinsic subtype & HER2BC vs non- HER2BC & 0.695 & $0.266-1.818$ & 0.459 & & & \\
\hline Intrinsic subtype & HRBC vs non-HRBC & 1.054 & $0.514-2.160$ & 0.886 & & & \\
\hline Pathological response & $p C R$ vs non-pCR & 0.611 & $0.279-1.336$ & 0.217 & 1.008 & $0.402-2.524$ & 0.987 \\
\hline TILS & High vs Low & 0.420 & $0.199-0.885$ & 0.022 & 4.785 & $1.169-19.582$ & 0.029 \\
\hline RCB-TILS & Positive vs Negative & 0.181 & $0.082-0.401$ & $<0.001$ & 0.048 & $0.012-0.188$ & $<0.001$ \\
\hline \multicolumn{8}{|l|}{$\operatorname{TNBC}(n=61)$} \\
\hline Age & $\leq 56$ vs $>56$ & 0.690 & $0.211-2.262$ & 0.541 & & & \\
\hline Menopause & Pre- vs Post- & 0.652 & $0.199-2.136$ & 0.480 & & & \\
\hline Tumor size $(\mathrm{cm})$ & $\leq 2$ vs $>2$ & 0.550 & $0.119-2.546$ & 0.444 & & & \\
\hline Lymph node status & Negative vs Positive & 0.942 & $0.203-4.359$ & 0.939 & & & \\
\hline Nuclear grade & $1-2$ vs 3 & 1.553 & $0.455-5.307$ & 0.482 & & & \\
\hline Ki67 (\%) & $\leq 14$ vs $>14$ & 0.739 & $0.216-2.526$ & 0.630 & & & \\
\hline Pathological response & $p C R$ vs non-pCR & 0.234 & $0.050-1.084$ & 0.063 & 0.270 & $0.030-2.466$ & 0.246 \\
\hline TILS & High vs Low & 0.177 & $0.054-0.583$ & 0.004 & 0.243 & $0.071-0.816$ & 0.023 \\
\hline RCB-TILs & Positive vs Negative & 0.099 & $0.029-0.343$ & $<0.001$ & 0.041 & $0.003-0.573$ & 0.018 \\
\hline \multicolumn{8}{|l|}{ HER2BC $(n=36)$} \\
\hline Age & $\leq 56$ vs $>56$ & 1.245 & $0.207-7.493$ & 0.811 & & & \\
\hline Menopause & Pre- vs Post- & 2.507 & $0.280-22.443$ & 0.411 & & & \\
\hline Tumor size (cm) & $\leq 2$ vs $>2$ & 0.693 & $0.081-6.302$ & 0.744 & & & \\
\hline Lymph node status & Negative vs Positive & 3.732 & $0.072-5.051$ & 0.414 & & & \\
\hline Nuclear grade & $1-2$ vs 3 & 0.043 & $0.011-5.216$ & 0.513 & & & \\
\hline Ki67 (\%) & $\leq 14$ vs $>14$ & 0.441 & $0.068-2.623$ & 0.364 & & & \\
\hline Pathological response & $p C R$ vs non-pCR & 0.482 & $0.078-2.847$ & 0.415 & 0.702 & $0.108-4.551$ & 0.710 \\
\hline TILs & High vs Low & 0.123 & $0.020-0.774$ & 0.026 & 0.134 & $0.020-0.879$ & 0.036 \\
\hline RCB-TILs & Positive vs Negative & 0.123 & $0.020-0.774$ & 0.026 & 0.134 & $0.020-0.879$ & 0.036 \\
\hline \multicolumn{8}{|l|}{$\operatorname{HRBC}(n=80)$} \\
\hline Age & $\leq 56$ vs $>56$ & 0.856 & $0.297-2.467$ & 0.773 & & & \\
\hline Menopause & Pre- vs Post- & 0.769 & $0.270-2.193$ & 0.623 & & & \\
\hline Tumor size $(\mathrm{cm})$ & $\leq 2$ vs $>2$ & 2.462 & $0.322-18.836$ & 0.386 & & & \\
\hline Lymph node status & Negative vs Positive & 3.682 & $0.151-10.382$ & 0.205 & & & \\
\hline Nuclear grade & $1-2$ vs 3 & 1.063 & $0.303-3.811$ & 0.930 & & & \\
\hline Ki67 (\%) & $\leq 14$ vs $>14$ & 0.602 & $0.212-1.738$ & 0.344 & & & \\
\hline Pathological response & $p C R$ vs non-pCR & 1.328 & $0.438-3.973$ & 0.614 & 2.123 & $0.667-6.750$ & 0.202 \\
\hline TILs & High vs Low & 0.992 & $0.311-3.165$ & 0.990 & 1.044 & $0.323-3.372$ & 0.949 \\
\hline RCB-TILS & Positive vs Negative & 0.258 & $0.071-0.932$ & 0.039 & 0.081 & $0.016-0.409$ & 0.002 \\
\hline
\end{tabular}

c.i confidence interval, TILs tumor-infiltrating lymphocytes, RCB residual cancer burden, TNBC triple-negative breast cancer, HER2BC human epidermal growth factor receptor 2-enriched breast cancer, $H R B C$ hormone receptor-positive breast cancer, $p C R$ pathological complete response 


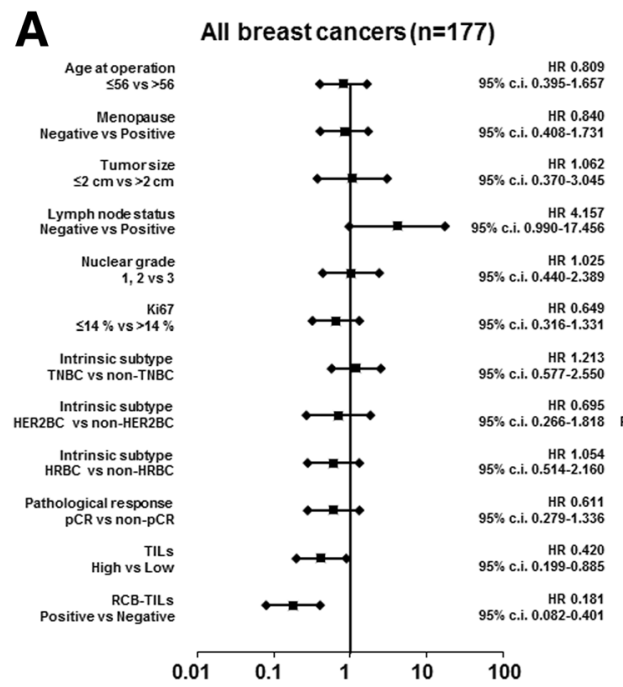

C

HER2-enriched breast cancers $(n=36)$

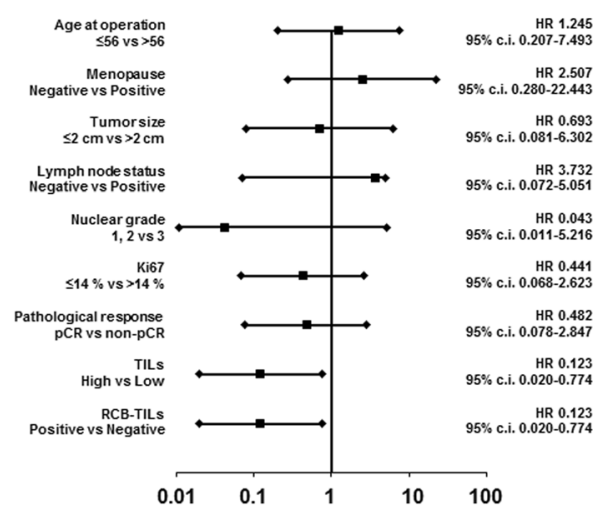

B Triple-negative breast cancers $(\mathrm{n}=61)$

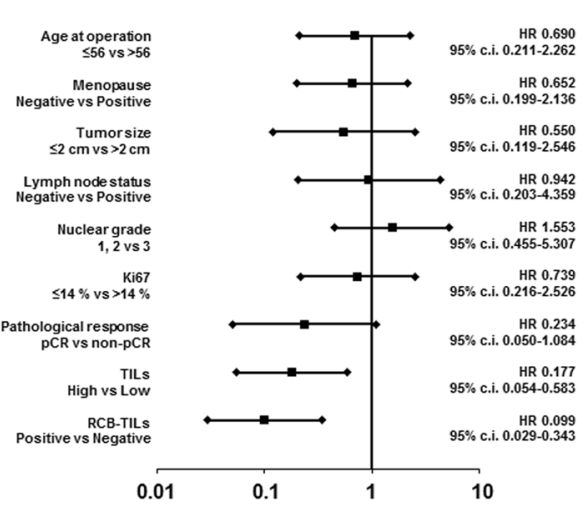

D Hormone receptor-positive breast cancer $(n=80)$

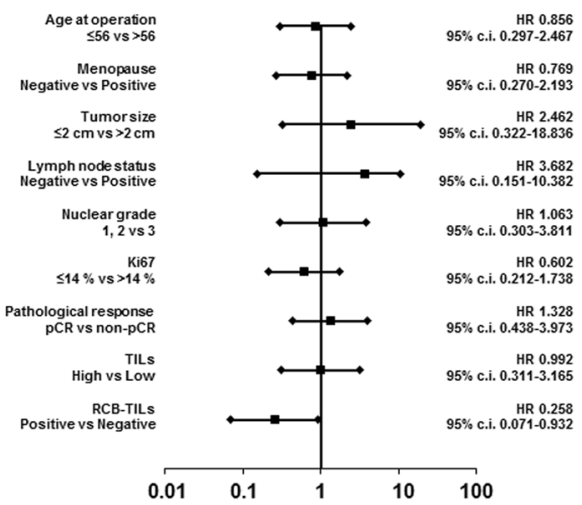

Fig. 2 Forest plots. Univariable analysis of patients with being RCB-TILs-positive found that this contributed significantly to prolonging DFS in all patients $(p<0.001$, hazard ratio $=0.181)(\mathbf{a})$, TNBC patients $(p<0.001$, hazard ratio $=0.099)(\mathbf{b})$, HER2BC patients $(p=0.026$, hazard ratio $=0.123)$ $(\mathbf{c})$, and HRBC patients $(p=0.039$, hazard ratio $=0.258)(\mathbf{d})$

who have achieved pCR as a result of NAC compared with non-pCR patients, and this is considered to be of major significance [32, 33]. However, although pCR does contribute to survival in highly malignant breast cancers such as TNBC and HER2BC, it has been shown that it does not provide an indicator of survival in the lowmalignancy subtype of HRBC [32, 34]. In the prediction of response to treatment, TILs evaluation is also only predictive of response to treatment with NAC in TNBC and HER2BC patients $[9,16,18]$. The subtype for which it is the most difficult to predict the response to treatment with NAC is thus HRBC, which is the most common. RCB evaluation after NAC, on the other hand, has been found to be useful for predicting survival in HRBC patients $[11,12]$. RCB-TILs, our proposed indicator, was useful for predicting survival to post-NAC recurrence in all subtypes.
TILs is regarded as a marker of subtypes with high immune activity, while pCR is considered to be a marker of subtypes with high cellular proliferation activity [7-9, 35]. In HRBC patients, RCB-TILs-positive patients had a significantly higher Ki67 value and higher pCR rate. In this study, the RCB-TILs-positive HRBC cases were found to have high immune activity and high cellular proliferation activity. When we combined the markers useful for the various different subtypes to create a new method of evaluation in terms of RCB-TILs, we were able to predict survival after NAC for patients with all of the various subtypes. We also showed that this is a more sensitive indicator than prediction by TILs alone. In the choice of additional treatment after NAC, RCB-TILs evaluation may thus contribute to treatment strategies that are neither excessive nor inadequate. However, this study had the limitations of being a retrospective 

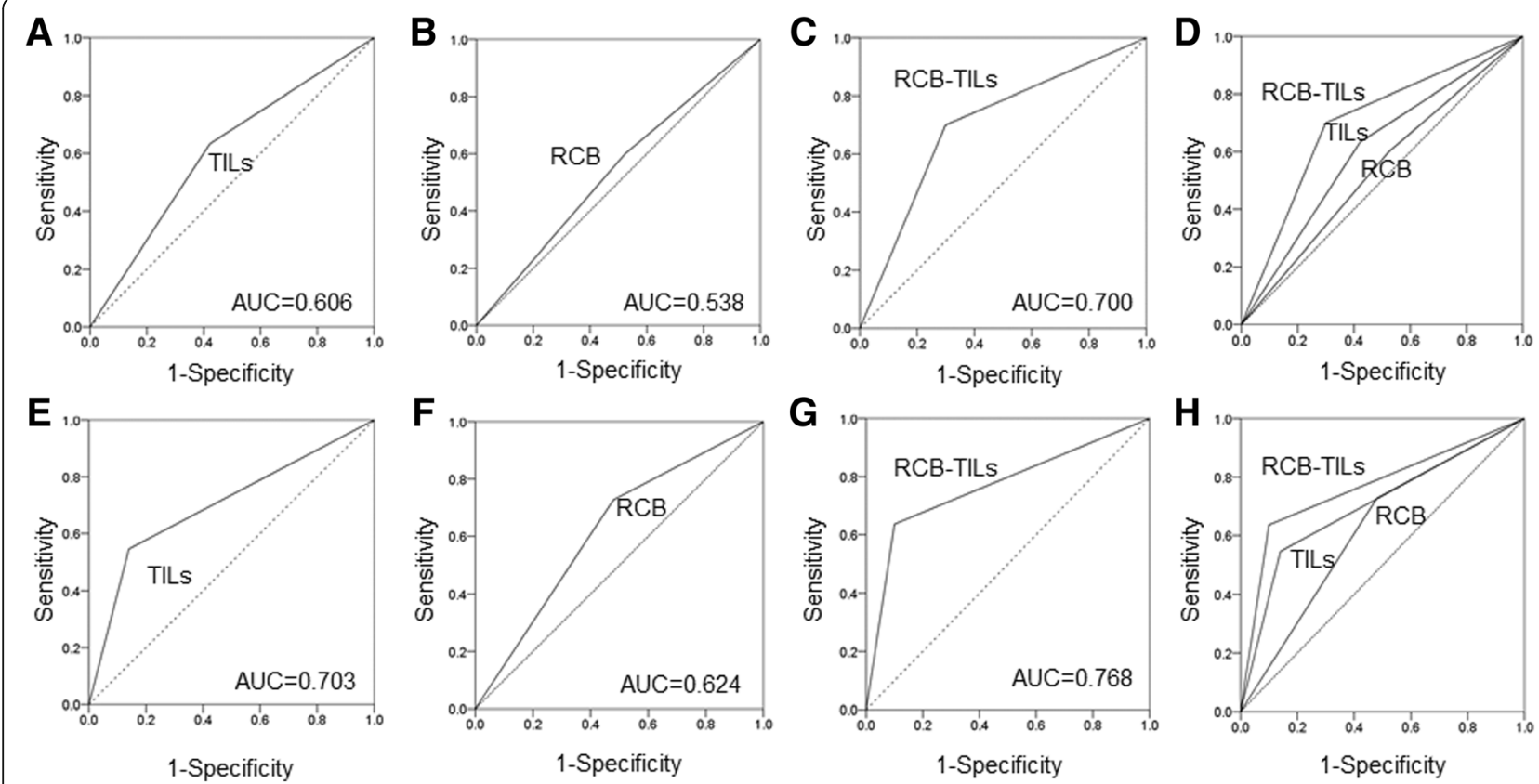

Fig. 3 On ROC curve analyses in all breast cancer and TNBC patients. ROC analysis showed that, for all breast cancer patients, the results for the RCB-TILS (AUC: 0.700) were better than those for the TILs (AUC: 0.606) and the RCB (AUC: 0.538) (a-d). ROC analysis for TNBC patients also found similar results (AUC: TILs $=0.703, \mathrm{RCB}=0.624, \mathrm{RCB}-\mathrm{TILs}=0.768)(\mathbf{e}-\mathbf{h})$
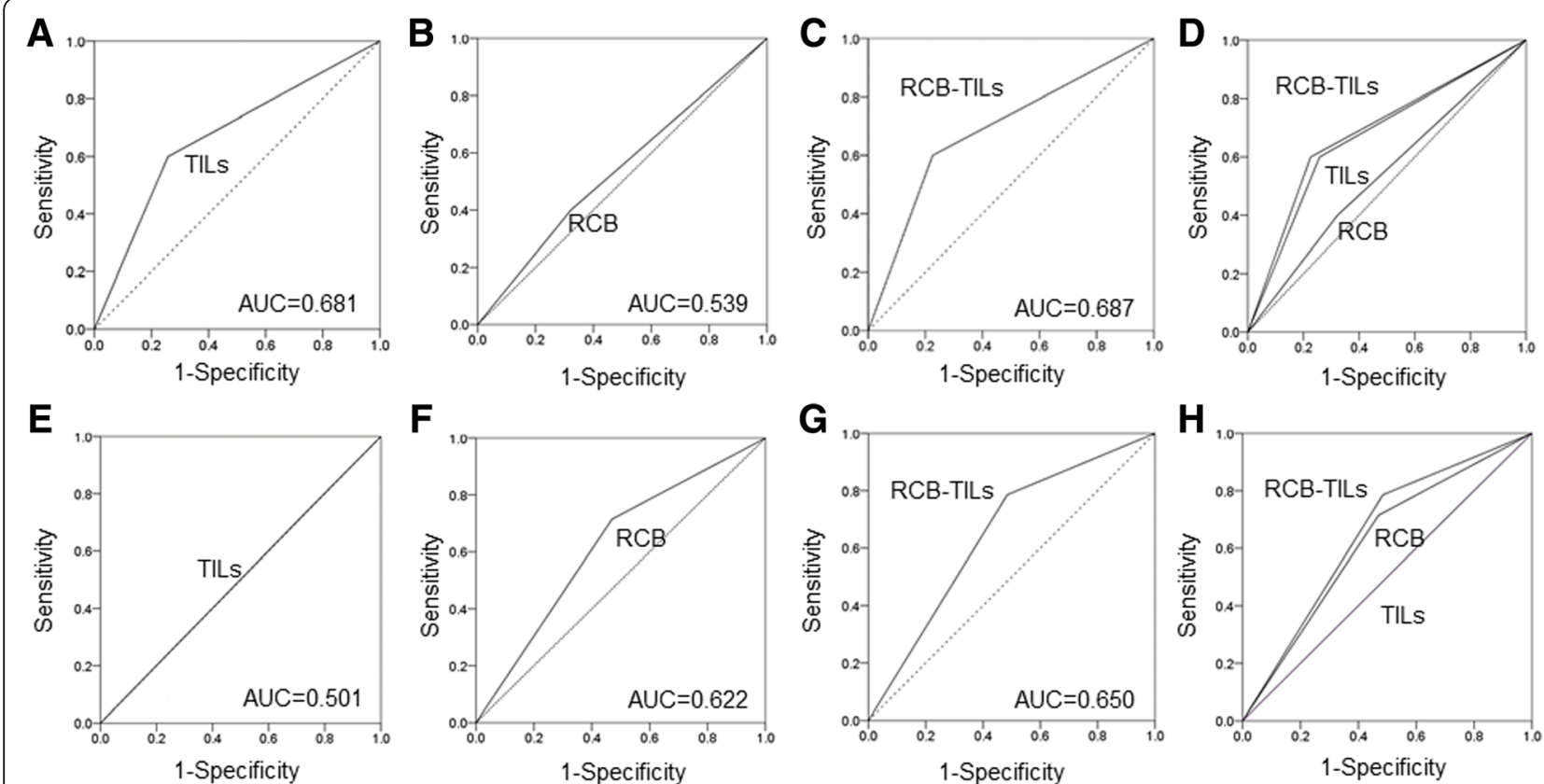

Fig. 4 On ROC curve analyses in HER2BC and HRBC patients. ROC analysis showed that, for HER2BC patients, the results for the RCB-TILs (AUC: 0.687) were better than those for the TILs (AUC: 0.681) and the RCB (AUC: 0.539) (a-d). ROC analysis for HRBC patients also found similar results (AUC: TILs $=0.501, \mathrm{RCB}=0.622, \mathrm{RCB}-\mathrm{TILS}=0.650)(\mathbf{e}-\mathbf{h})$ 
investigation and of differences in adjuvant therapy after NAC. Clinical trials of CREAT-X and other adjuvant therapies after NAC are currently being reported [36]. It is to be hoped that such clinical trials will also investigate the validity of RCB-TILs for predicting survival after NAC.

There are some subtypes of HRBC for which endocrine therapy is relatively ineffective. In this study, all HRBC patients were treated with postoperative endocrine therapy. However, RCB-TILs-negative patients had a high rate of recurrence, suggesting that RCB-TILs may provide a marker for predicting the response to endocrine therapy. A new treatment strategy is conceivable whereby RCBTILs-positive HRBC patients undergo conventional endocrine therapy after NAC while additional chemotherapy is chosen for those who are RCB-TILs-negative.

\section{Conclusions}

The results of the present study suggest that RCB-TILs is a significant predictor for breast cancer recurrence after NAC and may be a more sensitive indicator than TILs alone.

\section{Additional file}

Additional file 1: Figure S1. Analysis of RCB-TILs status and outcome in breast cancer (Overall Survival, OS). OS was significantly longer for RCB-TILs-positive patients than for RCB-TILs-negative patients in all patients ( $p=0.005$, log-rank) $(\mathbf{A})$ and TNBC patients $(p<0.001$, log-rank) $(\mathbf{B})$, but the difference was not significant for HER2BC patients ( $p=0.585$, log-rank) (C) or HRBC patients ( $p=0.128$, log-rank) (D). (ZIP $154 \mathrm{~kb})$

\section{Abbreviations \\ AUC: Area under the curve; cil: Confidence interval; CNB: Core needle biopsy; $C$ : Computed tomography; DFS: Disease-free survival; ER: Estrogen receptor; HE: Hematoxylin and eosin; HER: Human epidermal growth factor receptor; HER2BC: HER2-enriched; HR: Hazard ratio; HRBC: Hormone receptor-positive breast cancer; NAC: Eoadjuvant chemotherapy; NSABP: National surgical adjuvant breast and bowel project; OS: Overall survival; pCR: Pathological complete response; PgR: Progesterone receptor; RCB: Residual cancer burden; RECIST: Response evaluation criteria in solid tumors; REMARK: Reporting recommendations for tumor marker prognostic studies; ROC: Receiver operating characteristic; TILs: Tumor-infiltrating lymphocytes; TNBC: Triple-negative breast cancer; TS: Training Set; UICC: Union for international cancer control; US: Ultrasonography; VS: Validation Set}

\section{Acknowledgements}

We thank Yayoi Matsukiyo and Tomomi Okawa (Department of Surgical Oncology, Osaka City University Graduate School of Medicine) for helpful advice regarding data management.

\section{Funding}

This study was supported in part by Grants-in Aid for Scientific Research (KAKENHI, Nos. 25,461,992 and 26,461,957) from the Ministry of Education, Science, Sports, Culture and Technology of Japan.

\section{Availability of data and materials}

The datasets supporting the conclusions of this article is included within the article.

\section{Authors' contributions}

All authors were involved in the preparation of this manuscript. YA collected the data, and wrote the manuscript. SK, WG, KTakada, KTakahashi, TH, SN, TT and NO performed the operation and designed the study. YA, SK and ST summarized the data and revised the manuscript. MOhsawa performed the pathological diagnosis. $\mathrm{HM}, \mathrm{KH}$ and MOhira substantial contribution to the study design, performed the operation, and revised the manuscript. All authors read and approved the final manuscript.

\section{Ethics approval and consent to participate}

Written informed consent was obtained from all subjects. This research conformed to the provisions of the Declaration of Helsinki in 2013. All patients were informed of the investigational nature of this study and provided their written, informed consent. The study protocol was approved by the Ethics Committee of Osaka City University (\#926).

\section{Consent for publication}

Not applicable.

\section{Competing interests}

The authors declare that they have no competing interests.

\section{Publisher's Note}

Springer Nature remains neutral with regard to jurisdictional claims in published maps and institutional affiliations.

\section{Author details}

'Department of Surgical Oncology, Asahi-machi, Abeno-ku, Osaka 545-8585, Japan. ${ }^{2}$ Department of Pharmacology, Asahi-machi, Abeno-ku, Osaka 545-8585, Japan. ${ }^{3}$ Department of Plastic and Reconstructive Surgery, 1-4-3 Asahi-machi, Abeno-ku, Osaka 545-8585, Japan. ${ }^{4}$ Department of Diagnostic Pathology, Osaka City University Graduate School of Medicine, 1-4-3 Asahi-machi, Abeno-ku, Osaka 545-8585, Japan.

Received: 9 June 2017 Accepted: 14 December 2017

Published online: 28 December 2017

References

1. Wolmark N, Wang J, Mamounas E, Bryant J, Fisher B. Preoperative chemotherapy in patients with operable breast cancer: nine-year results from National Surgical Adjuvant Breast and bowel project B-18. J Natl Cancer Inst Monogr. 2001;30:96-102.

2. van der Hage JA, van de Velde CJ, Julien JP, Tubiana-Hulin M, Vandervelden C, Duchateau L. Preoperative chemotherapy in primary operable breast cancer: results from the European Organization for Research and Treatment of cancer trial 10902. J Clin Oncol. 2001;19(22):4224-37.

3. Mayer EL, Carey LA, Burstein HJ. Clinical trial update: implications and management of residual disease after neoadjuvant therapy for breast cancer. Breast Cancer Res. 2007;9(5):110.

4. Sachelarie I, Grossbard ML, Chadha M, Feldman S, Ghesani M, Blum RH. Primary systemic therapy of breast cancer. Oncologist. 2006;11(6):574-89.

5. Bear HD, Anderson S, Brown A, Smith R, Mamounas EP, Fisher B, Margolese $\mathrm{R}$, Theoret $\mathrm{H}$, Soran A, Wickerham DL, et al. The effect on tumor response of adding sequential preoperative docetaxel to preoperative doxorubicin and cyclophosphamide: preliminary results from National Surgical Adjuvant Breast and bowel project protocol B-27. J Clin Oncol. 2003;21(22):4165-74.

6. Henderson IC, Berry DA, Demetri GD, Cirrincione $C T$, Goldstein $L$, Martino $S$, Ingle JN, Cooper MR, Hayes DF, Tkaczuk KH, et al. Improved outcomes from adding sequential Paclitaxel but not from escalating doxorubicin dose in an adjuvant chemotherapy regimen for patients with node-positive primary breast cancer. J Clin Oncol. 2003;21(6):976-83.

7. Kaufmann M, Hortobagyi GN, Goldhirsch A, Scholl S, Makris A, Valagussa P, Blohmer JU, Eiermann W, Jackesz R, Jonat W, et al. Recommendations from an international expert panel on the use of neoadjuvant (primary) systemic treatment of operable breast cancer: an update. J Clin Oncol. 2006;24(12): 1940-9.

8. Mukai H, Arihiro K, Shimizu C, Masuda N, Miyagi Y, Yamaguchi T, Yoshida T. Stratifying the outcome after neoadjuvant treatment using pathological response classification by the Japanese breast cancer society. Breast Cancer. 2016;23(1):73-7. 
9. Savas P, Salgado R, Denkert C, Sotiriou C, Darcy PK, Smyth MJ, Loi S. Clinical relevance of host immunity in breast cancer: from TILs to the clinic. Nat Rev Clin Oncol. 2016;13(4):228-41.

10. Dieci MV, Criscitiello C, Goubar A, Viale G, Conte P, Guarneri V, Ficarra G, Mathieu MC, Delaloge S, Curigliano G, et al. Prognostic value of tumor-infiltrating lymphocytes on residual disease after primary chemotherapy for triple-negative breast cancer: a retrospective multicenter study. Ann Oncol. 2014;25(3):611-8.

11. Symmans WF, Peintinger F, Hatzis C, Rajan R, Kuerer H, Valero V, Assad L, Poniecka A, Hennessy B, Green M, et al. Measurement of residual breast cancer burden to predict survival after neoadjuvant chemotherapy. J Clin Oncol. 2007;25(28):4414-22.

12. Sheri A, Smith IE, Johnston SR, A'Hern R, Nerurkar A, Jones RL, Hills M, Detre S, Pinder SE, Symmans WF, et al. Residual proliferative cancer burden to predict long-term outcome following neoadjuvant chemotherapy. Ann Oncol. 2015;26(1):75-80

13. Hanahan D, Weinberg RA. Hallmarks of cancer: the next generation. Cell. 2011;144(5):646-74.

14. Dougan M, Dranoff G. Immune therapy for cancer. Annu Rev Immunol. 2009;27:83-117.

15. Adams S, Gray RJ, Demaria S, Goldstein L, Perez EA, Shulman LN, Martino S, Wang M, Jones VE, Saphner TJ, et al. Prognostic value of tumor-infiltrating lymphocytes in triple-negative breast cancers from two phase III randomized adjuvant breast cancer trials: ECOG 2197 and ECOG 1199. J Clin Oncol. 2014; 32(27):2959-66.

16. Denkert C, von Minckwitz G, Brase JC, Sinn BV, Gade S, Kronenwett R, Pfitzner BM, Salat C, Loi S, Schmitt WD, et al. Tumor-infiltrating lymphocytes and response to neoadjuvant chemotherapy with or without carboplatin in human epidermal growth factor receptor 2-positive and triple-negative primary breast cancers. J Clin Oncol. 2015;33(9):983-91.

17. Loi S, Sirtaine N, Piette F, Salgado R, Viale G, Van Eenoo F, Rouas G, Francis $P$, Crown JP, Hitre $E$, et al. Prognostic and predictive value of tumor-infiltrating lymphocytes in a phase III randomized adjuvant breast cancer trial in node-positive breast cancer comparing the addition of docetaxel to doxorubicin with doxorubicin-based chemotherapy: BIG 02-98. J Clin Oncol. 2013;31(7):860-7.

18. Loi S, Michiels S, Salgado R, Sirtaine N, Jose V, Fumagalli D, KellokumpuLehtinen PL, Bono P, Kataja V, Desmedt C, et al. Tumor infiltrating lymphocytes are prognostic in triple negative breast cancer and predictive for trastuzumab benefit in early breast cancer: results from the FinHER trial. Ann Oncol. 2014;25(8):1544-50.

19. Asano Y, Kashiwagi S, Goto W, Kurata K, Noda S, Takashima T, Onoda N, Tanaka S, Ohsawa M, Hirakawa K. Tumour-infiltrating CD8 to FOXP3 lymphocyte ratio in predicting treatment responses to neoadjuvant chemotherapy of aggressive breast cancer. Br J Surg. 2016;103(7):845-54.

20. Ingold Heppner B, Untch M, Denkert C, Pfitzner BM, Lederer B, Schmitt W Eidtmann H, Fasching PA, Tesch H, Solbach C, et al. Tumor-infiltrating lymphocytes: a predictive and prognostic biomarker in Neoadjuvant-treated HER2-positive breast cancer. Clin Cancer Res. 2016;22(23):5747-54.

21. Greene FL, Sobin LH. A worldwide approach to the TNM staging system: collaborative efforts of the AJCC and UICC. J Surg Oncol. 2009;99(5):269-72.

22. Asano Y, Kashiwagi S, Onoda N, Kurata K, Morisaki T, Noda S, Takashima T, Ohsawa M, Kitagawa S, Hirakawa K. Clinical verification of sensitivity to preoperative chemotherapy in cases of androgen receptor-expressing positive breast cancer. Br J Cancer. 2016;114(1):14-20.

23. Goldhirsch A, Wood WC, Coates AS, Gelber RD, Thurlimann B, Senn HJ, Panel M. Strategies for subtypes-dealing with the diversity of breast cancer: highlights of the St. Gallen international expert consensus on the primary therapy of early breast cancer 2011. Ann Oncol. 2011;22(8):1736-47.

24. Mauri D, Pavlidis N, loannidis JP. Neoadjuvant versus adjuvant systemic treatment in breast cancer: a meta-analysis. J Natl Cancer Inst. 2005;97(3):188-94.

25. Mieog JS, van der Hage JA, van de Velde CJ. Preoperative chemotherapy for women with operable breast cancer. Cochrane Database Syst Rev. 2007;2:CD005002.

26. Buzdar AU, Valero V, Ibrahim NK, Francis D, Broglio KR, Theriault RL, Pusztai L, Green MC, Singletary SE, Hunt KK, et al. Neoadjuvant therapy with paclitaxel followed by 5-fluorouracil, epirubicin, and cyclophosphamide chemotherapy and concurrent trastuzumab in human epidermal growth factor receptor 2-positive operable breast cancer: an update of the initial randomized study population and data of additional patients treated with the same regimen. Clin Cancer Res. 2007:13(1):228-33.
27. Eisenhauer EA, Therasse P, Bogaerts J, Schwartz LH, Sargent D, Ford R, Dancey J, Arbuck S, Gwyther S, Mooney M, et al. New response evaluation criteria in solid tumours: revised RECIST guideline (version 1.1). Eur J Cancer. 2009:45(2):228-47.

28. Kashiwagi S, Asano Y, Goto W, Takada K, Takahashi K, Noda S, Takashima T, Onoda N, Tomita S, Ohsawa M, et al. Use of tumor-infiltrating lymphocytes (TILs) to predict the treatment response to eribulin chemotherapy in breast cancer. PLoS One. 2017;12(2):e0170634.

29. Salgado R, Denkert C, Demaria S, Sirtaine N, Klauschen F, Pruneri G, Wienert S, Van den Eynden G, Baehner FL, Penault-Llorca F, et al. The evaluation of tumor-infiltrating lymphocytes (TILs) in breast cancer: recommendations by an international TILs working group 2014. Ann Oncol. 2015;26(2):259-71.

30. Ono M, Tsuda H, Shimizu C, Yamamoto S, Shibata T, Yamamoto H, Hirata T, Yonemori K, Ando M, Tamura K, et al. Tumor-infiltrating lymphocytes are correlated with response to neoadjuvant chemotherapy in triple-negative breast cancer. Breast Cancer Res Treat. 2012;132(3):793-805.

31. Mao Y, Qu Q, Zhang Y, Liu J, Chen X, Shen K. The value of tumor infiltrating lymphocytes (TILs) for predicting response to neoadjuvant chemotherapy in breast cancer: a systematic review and meta-analysis. PLoS One. 2014; 9(12):e115103.

32. Cortazar P, Zhang L, Untch M, Mehta K, Costantino JP, Wolmark N, Bonnefoi $H$, Cameron D, Gianni L, Valagussa $P$, et al. Pathological complete response and long-term clinical benefit in breast cancer: the CTNeoBC pooled analysis. Lancet. 2014;384(9938):164-72.

33. Rastogi P, Anderson SJ, Bear HD, Geyer CE, Kahlenberg MS, Robidoux A, Margolese RG, Hoehn JL, Vogel VG, Dakhil SR, et al. Preoperative chemotherapy: updates of National Surgical Adjuvant Breast and bowel project protocols B-18 and B-27. J Clin Oncol. 2008;26(5):778-85.

34. Houssami N, Macaskill P, von Minckwitz G, Marinovich ML, Mamounas E. Meta-analysis of the association of breast cancer subtype and pathologic complete response to neoadjuvant chemotherapy. Eur J Cancer. 2012; 48(18):3342-54.

35. von Minckwitz G, Untch M, Blohmer JU, Costa SD, Eidtmann H, Fasching PA Gerber B, Eiermann W, Hilfrich J, Huober J, et al. Definition and impact of pathologic complete response on prognosis after neoadjuvant chemotherapy in various intrinsic breast cancer subtypes. J Clin Oncol. 2012;30(15):1796-804.

36. Masuda N, Lee SJ, Ohtani S, Im YH, Lee ES, Yokota I, Kuroi K, Im SA, Park BW, Kim SB, et al. Adjuvant Capecitabine for breast cancer after preoperative chemotherapy. N Engl J Med. 2017:376(22):2147-59.

\section{Submit your next manuscript to BioMed Central and we will help you at every step:}

- We accept pre-submission inquiries

- Our selector tool helps you to find the most relevant journal

- We provide round the clock customer support

- Convenient online submission

- Thorough peer review

- Inclusion in PubMed and all major indexing services

- Maximum visibility for your research

Submit your manuscript at www.biomedcentral.com/submit
) Biomed Central 